OPEN ACCESS

Edited by:

Miao Yu,

Chongqing University, China

Reviewed by:

Yancheng Li,

University of Technology Sydney,

Australia

Xinglong Gong Gong,

University of Science and Technology

of China, China

*Correspondence:

Ying-Qing Guo

gyingqing@126.com

Specialty section:

This article was submitted to

Smart Materials,

a section of the journal

Frontiers in Materials

Received: 17 March 2018

Accepted: 31 July 2018

Published: 21 August 2018

Citation:

Guo $Y$-Q, Sun C-L, Xu Z-D and Jing $X$ (2018) Preparation and Tests of MR

Fluids With Cl Particles Coated With

MWNTs. Front. Mater. 5:50

doi: $10.3389 /$ fmats.2018.00050

\section{Preparation and Tests of MR Fluids With CI Particles Coated With MWNTs}

\author{
Ying-Qing Guo ${ }^{1 *}$, Chun-Li Sun ${ }^{2}$, Zhao-Dong $X u^{2}$ and Xingjian Jing ${ }^{3}$ \\ ${ }^{1}$ Mechanical and Electronic Engineering College, Nanjing Forestry University, Nanjing, China, ${ }^{2}$ Key Laboratory of C\&PC \\ Structures of the Ministry of Education, Southeast University, Nanjing, China, ${ }^{3}$ Department of Mechanical Engineering, The \\ Hong Kong Polytechnic University, Hong Kong, Hong Kong
}

The magnetorheological (MR) fluid is a typical smart material, whose shear yield stress can be adjusted through changing the strength of external magnetic field, and the changing process only takes a few milliseconds. The MR fluid is composed of micro/nanometer ferromagnetic particles, carrier fluids, and some additives. Among them, the performance of ferromagnetic particles will mainly affect the sedimentation stability and the magnetic saturation of the MR fluid. Therefore, the ferromagnetic particles are expected to have characteristics of both low density and high magnetism. In this paper, the multi-walled carbon nanotubes (MWNTs) were adopted to coat on the carbonyl iron $(\mathrm{Cl})$ particles with grafting technology using ultrasonication and mechanical stirring. The coated $\mathrm{Cl}$ particles with perfect core-shell structure were developed and the influence of the dosages of grafting agent and MWNTs were tested. And then, MR fluids with $\mathrm{Cl}$ particles coated with MWNTs were established and the coating effect was studied through surface topography particle density, and magnetic properties of composite magnetic particles and stability tests of the prepared MR fluids. The results showed that although the magnetic saturation of the prepared MR fluids with $\mathrm{Cl}$ particles coated with MWNTs would reduce slightly, the particles density and the adsorption force between the particles were decreased effectively, which are both advantageous to the improvement of the sedimentation stability of MR fluids.

\footnotetext{
Keywords: magnetorheological fluids, the carbonyl iron particles, the multi-walled carbon nanotubes, coating effect, property tests
}

\section{INTRODUCTION}

Magnetorheological (MR) fluid is a kind of typical smart material with unique magnetic rheological properties characterized by a reversible change in viscosity and yield stress under magnetic field (Kim et al., 2013), which has been widely applied in many fields, such as sealing (Mitamura et al., 2008), target drug delivery (Kim and Kim, 2003; Oh and Park, 2011), lubrication (Arruebo et al., 2007), removal of water pollutants (Goldowsky, 1980), dampers (Olabi and Grunwald, 2007; Ferroudj et al., 2013; Zong et al., 2013; Zhu et al., 2018), brakes (Mangal and Kumar, 2015) etc. Rabinow (1948) prepared the original MR fluid consisting of magnetic particles and carrier liquid in 1948. Xu et al. (2003) reported that MR fluids can transform between solid and liquid due to the existence of the magnetic particles; specifically, the iron particles are magnetized and MR fluids can be changed rapidly from liquid to solid in the presence of a magnetic field, while 
regaining their liquid state when the magnetic field is removed. The instantaneous conversion of solid and liquid can be used to adjust the stiffness and the damping of MR fluids. For decades, many researchers have devoted themselves to the study of MR fluids. Currently, the ferromagnetic particles adopted for preparing MR fluids are mainly carbonyl iron (CI) particles with diameter of generally $0.1-10 \mu \mathrm{m}$, and the volume fraction of ferromagnetic particles is generally $20-40 \%$. However, the density of a ferromagnetic particle is much larger than that of the carrier fluids, which would definitely lead to obvious sedimentation, restrict the rheological properties of MR fluids and reduce the adjustability and shock absorption capacity of MR devices. Therefore, many researchers have proposed various methods to prepare high performance MR fluids with better sedimentation stability (Tian et al., 2016). Thomas (1966) obtained cobalt element powder by heating the carbonyl compounds and took cobalt powders as magnetic particles to prepare MR fluids. Kormann (Kormann et al., 1996) developed MR fluids consisting of magnetic nanometer particles which greatly improved shear yield strength and shortened response time. Foister (1997) added two kinds of iron particles with different sizes into the carrier fluids, indicating that the shear yield stress of MR fluids had improved a lot through this method. Ulicny and Mance (2004) studied the antioxidation property of MR fluids and proposed a method of coating with a layer of nickel on the surface of the ferromagnetic particles to improve the long-term oxidation resistance of MR fluids. Cheng et al. (2009) employed $\mathrm{N}$-glucose ethylene diamine triacetic acid, which can form a network coating CI particles to reduce the sedimentation rate via hydrogen bonds in water. Du et al. (2010) adopted the hydrophilic-lipophilic balance theory to choose the surfactant and discovered that surfactants could enhance the sedimentation stability of MR fluids, while decrease the magnetic properties of the particles or the rheological properties of MR fluids to some degree. Kim and Choi (2011) employed polymeric compounds as a carrier fluid and found that dispersing CI particles in a mixture of polyisobutylene/polybutene (PIB/PB) instead of the mineral oil could improve the stability and rheological properties of MR fluids. Sedlacik et al. (2011) coated fluorine bonds on the surface of CI particles by exposing CI particles to argon and octafluorocyclobutane plasma and concluded that the MR fluid based on plasma modified CI particles present better stability than that with pure CI particles. Dong et al. (2012) introduced a novel MR fluid to improve the MR properties by dispersing $\mathrm{Fe}_{76} \mathrm{Cr}_{2} \mathrm{Mo}_{2} \mathrm{Sn}_{2} \mathrm{P}_{10} \mathrm{~B}_{2} \mathrm{C}_{2} \mathrm{Si}_{4}$ amorphous alloy particles in silicon oil and discovered that the MR effect and sedimentation stability properties of MR fluids contained amorphous have improved significantly at lower field intensities. Kim and Choi (2011) prepared the polymeric solution by solving PEO in distilled water and acquired an MR fluid from the distribution of carbonyl iron in a polymeric solution, however, the mismatching of magnetic particles and the carrier liquid still remained to be overcome in order to make considerable breakthroughs in improving the sedimentation stability of MR fluids.

Currently, it has become a hot topic by adopting the composite particles to prepare MR fluids in order to improve the stability and rheological properties of MR fluids. Cho et al.
(2004) obtained the low-density magnetic particles by coating CI particles with polymethyl methacrylate (PMMA). Qiao et al. (2010) prepared the polyethyene glycol (PEG)-coated $\mathrm{Fe}_{3} \mathrm{O}_{4}$ ferrofluids by suspending the PEG-coated $\mathrm{Fe}_{3} \mathrm{O}_{4}$ nanoparticles in an oligomeric PEG-400 carrier liquid, and investigated their magnetorheological steady flow behavior. Jiang et al. (2010) adopted coated poly (methyl methacrylate) (PMMA) to coat on the surface of CI particles in order to obtain composite CIPMMA particles and discovered the prepared MR fluid based on CI-PMMA particles exhibited better sedimentation stability. Lopez-Lopez et al. (2012) prepared the MR fluid based on the $50 \%$ volume iron particles coated with silica and concluded that $50 \%$ volume is the upper limit of particles concentration in such MR fluids. Liu et al. (2012) studied the recent researches on the synthesis of polymeric composite magnetic particles as dispersed phase in MR fluids and found that both the surface morphology and mass ratio of the polymeric layer have influence on the MR effect and stability of MR fluids. Sutrisno et al. (2013) prepared an MR fluid with high viscosity based on grafted poly-iron particles, exhibiting excellent thermosoxidative stability as well as nearly constant viscosity. Mrlik et al. (2013) adopted cholesteryl chloroformate to coat on the surface of CI particles and discovered that the sedimentation rate of MR fluids based on the prepared composite particles has greatly decreased due to better compatibility between the particles and silicon oil. In general, the adoption of composite particles can improve the stability of MR fluids greatly; however, there are still many deficiencies remained to be improved, i.e., the coating layers are not compacted and stable enough, which would lead to unsatisfactory reduction in particles density, resulting in unexpected increase in sedimentation rate. Therefore, it is an urgent to investigate and choose reasonable composite particles as well as preparation methods.

In this paper, MWNTs were adopted to coat on CI particles through the grafting technology to reduce the density of CI particles and the influence of the dosages of grafting agent and MWNTs were tested. Besides, MR fluids with CI particles coated with MWNTs were prepared and the coating effect was studied through surface topography analysis, particle density, and magnetic properties of composite magnetic particles. Furthermore, the redispersibility property and sedimentation rates were observed to confirm the effect of the coated CI particles in improving the stability of MR fluids.

\section{PREPARATION OF MR FLUIDS}

\section{Materials and Equipment}

Original CI particles (tap-density: $4.2 \mathrm{~g} / \mathrm{cm}^{3}$, mean size: $1.9 \mu \mathrm{m}$ ) coated with MWNTs (the tube diameter: 8-15 nm) are adopted as magnetic particles by grafting process with $\mathrm{P}$-aminobenzoic acid (PABA). Methyl silicone oil (the density: $0.963 \mathrm{~g} / \mathrm{cm}^{3}$, the dynamic viscosity: $0.482 \mathrm{~Pa} \cdot \mathrm{s}$ ) is employed as the carrier liquid in MR fluids. And some additives are used as dispersant, lubricant and anti-sedimentation agent, which includes liquid paraffin, graphite and bentonite. Meanwhile, distilled water is used as solvent in the whole process of the experiment. As for the main equipment adopted in this paper, the electric mixer is adopted 
to get the solution mixed uniformly, and the ultrasonic cleaner is responsible for getting the solution dispersed evenly because ultrasonic dispersion can destroy Coulomb force and van der Waals force between small particles. Additionally, the vacuum oven contributes to the drying of the obtained mixed liquid with appropriate temperature of $70^{\circ} \mathrm{C}$.

\section{Preparation of CI Particles Coated With MWNTs}

The MWNTs are a kind of allotropes of carbon whose tube diameters are from nanometers to decades of nanometer with filament shape and micron length on average. Besides, MWNTs are lightweight materials with density about $2 \mathrm{~g} / \mathrm{cm}^{3}$ at room temperature, which is half of the tap-density of CI particles $\left(4.2 \mathrm{~g} / \mathrm{cm}^{3}\right)$. Therefore, the density of CI particles would be greatly reduced when coated with MWNTs on the surface, accompanying with a slight or even negligible decrease in the magnetism of coated particles due to the presence of iron catalyzer in MWNTs. Consequently, the MWNTs are adopted as coating materials to prepare the composite magnetic particles.

It is an essential process to form a MWNTs layer on the surface of CI particles to prepare the composite magnetic particles. In this study, the surface grafting technology adopted to form the MWNTs layer, which can be described as making a grafting reaction between the active groups on the surface of the particles. To be specific, the organics with suitable monomer are chosen in the in-situ polymerization, and the polymer materials are coated on the surface of particles, i.e., surface modification. A grafting agent named para aminobenzoic acid (PABA) is employed in the grafting process which has two groups, hydroxy $(-\mathrm{OH})$ and amidogen $(-\mathrm{NH} 2)$. The hydroxy $(-\mathrm{OH})$ can combine with the carbonyl $(-\mathrm{C}=\mathrm{O})$ existing in the surface of $\mathrm{CI}$ particles and the amidogen $\left(-\mathrm{NH}_{2}\right)$ can combine with the carboxyl $(-\mathrm{COOH})$ in the MWNTs under certain conditions, contributing to the presentation of the results that the MWNTs tightly coated on the surface of CI particles, as shown in Figure 1.

The grafting process is not spontaneous, so the ultrasonication and the mechanical stirring are adopted simultaneously to induce

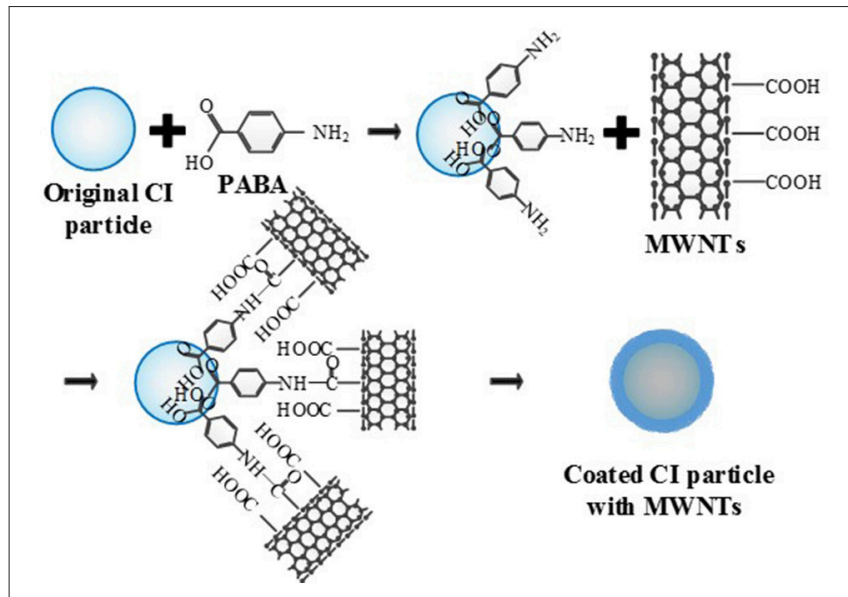

FIGURE 1 | The schematic diagram of the grafting process. the grafting process and enhance the coating effect. Ultrasonic treatment can induce PABA to build bridges between CI particles and MWNTs in order to modify their contact surfaces and form interaction functional groups between non-covalent bonds. Of course, there are two notes that must be emphasized. First one, the ultrasonication and the mechanical stirring the ultrasonic intensity should be controlled in a reasonable range, which is commonly set between 300 and $400 \mathrm{~W}$. If the ultrasonic intensity was set too low, it is difficult to cause the grafting process, otherwise it the formed MWNTs layer is destroyed. Second one, the compactness of coating is closely related to the temperature, when the temperature higher than $70^{\circ} \mathrm{C}$ the MWNTs are prone to intertwined with each other, and when the temperature is lower than $50^{\circ} \mathrm{C}$ it is difficult for the occurrence of the grafting reaction, so the temperature is usually set between 50 and $70^{\circ} \mathrm{C}$.

The preparation process of CI particles coated with MWNTs is as described below, as shown in Figure 2. Step one, PABA are dispersed into the distilled water via water-bath heating for $1-2 \mathrm{~h}$ with the temperature of $60^{\circ} \mathrm{C}$. Step two, the CI particles are dissolved in the mixed solution under the mild ultrasonic dispersion for about 15-20 min, and then under the mild ultrasonic wave issued by the ultrasonic cleaner with the frequency of $60 \mathrm{~Hz}$ the MWNTs are added into the above solution with mechanical stirring by the electric mixer for $\sim 3-4 \mathrm{~h}$. Step three, in general the obtained mixed liquid is dried by vacuum oven with the temperature of $70^{\circ} \mathrm{C}$ and the negative vacuum degree, after that the dried powder is ground and sieved into particles by 100 mesh sieve. As a matter of fact, the higher the vacuum degree is, the better the effect of the drying will be, and it mostly depends on the available vacuum degree provided by the vacuum oven adopted.

\section{Preparation Process of MR Fluids}

There are two major processes when preparing MR fluids, one is about the preparation of CI particles coated with MWNTs; the other is regarding the mixing of magnetic particles, carrier liquid, and additives. The detailed process is as shown in Figure 3. First of all, the CI particles coated with MWNTs are added into the carrier liquid, and then stirred by the electric mixer for $\sim 2-$ $3 \mathrm{~h}$ to make the liquid disperse uniformly. Afterward, every $2 \mathrm{~h}$ different kinds of additives including dispersant, lubricant, and anti-sedimentation agent are added quantitatively in sequence, at the same time, make the liquid keep continuous stirring.

\section{TESTS RESULTS AND ANALYSIS}

According to the preparation process of CI Particles coated with MWNTs, there were six kinds of coated CI particles with different dosages of grafting agent and MWNTs that were prepared. Then, series performance tests which included surface topography, the particle density and magnetic properties were carried out with variable-controlling approach step by step to determine the best component proportion, which contributed to the preparation of MR fluids with better performance. Finally, sedimentation tests on the MR fluids were conducted to confirm the coating effect. 


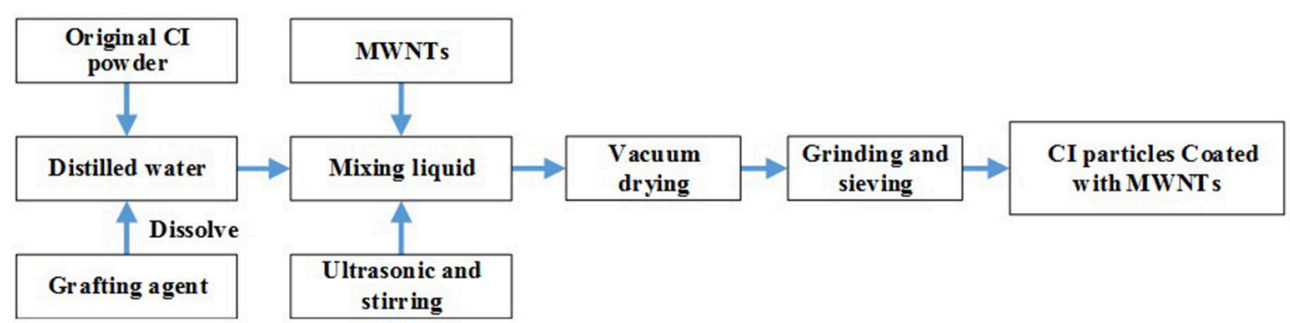

FIGURE 2 | Preparation process of $\mathrm{Cl}$ particles coated with MWNTs.

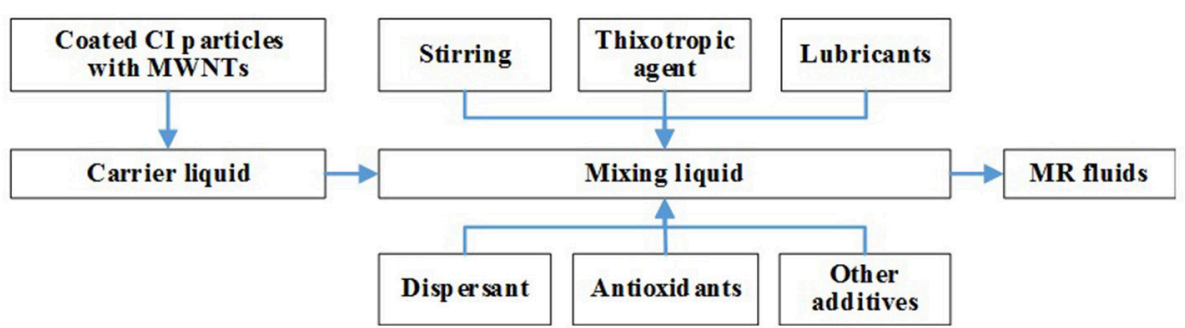

FIGURE 3 | Preparation process of MR fluids
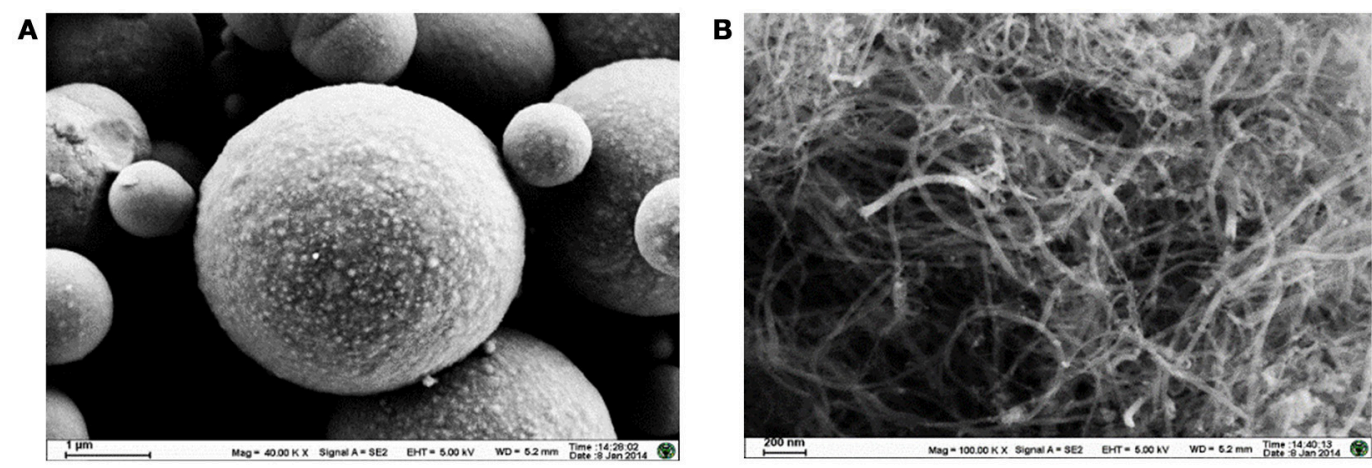

FIGURE 4 | The electron micrograps of (A) original Cl particles and (B) MWNTs.

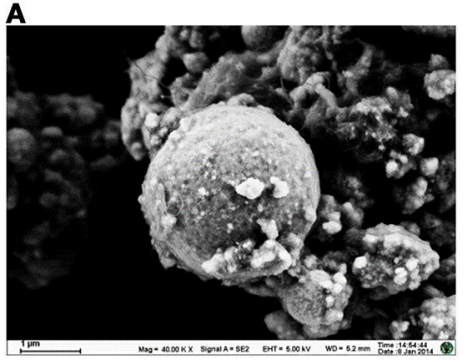

Sample A1

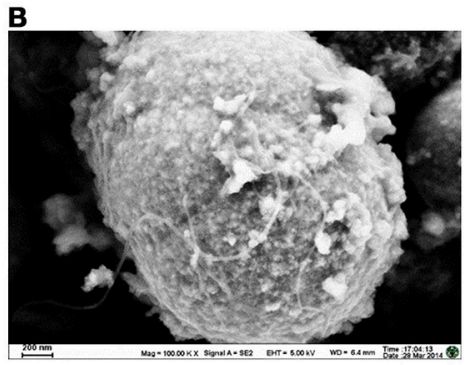

Sample A2

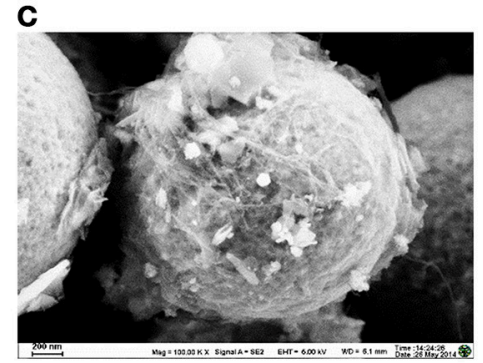

Sample A3

FIGURE 5 | The electron micrographs of composite particles with different dosages of grafting agent. 


\section{A}

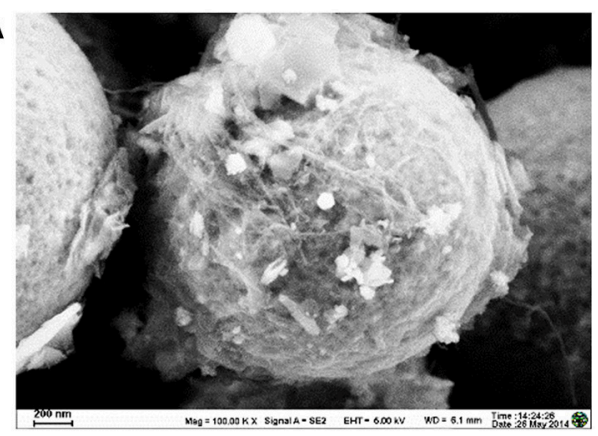

Sample A3

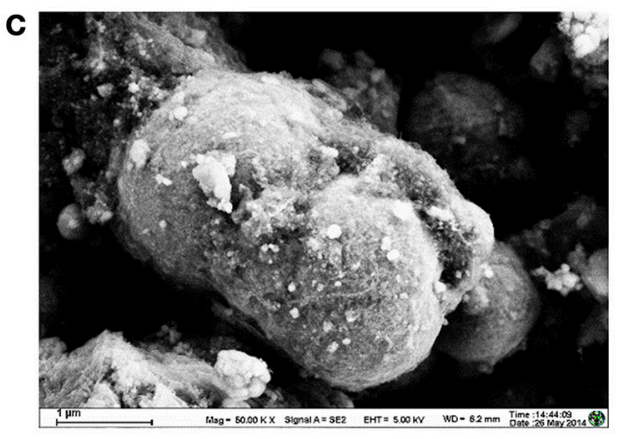

Sample B2

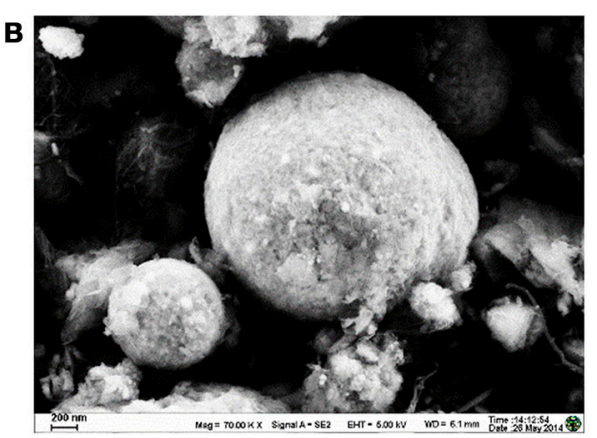

Sample B1

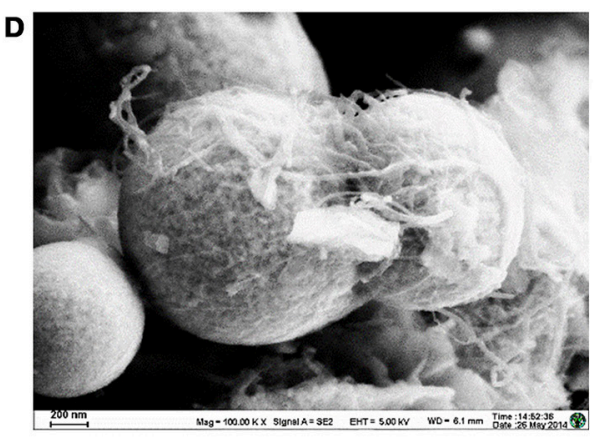

Sample B3

FIGURE 6 | The electron micrographs of composite particles with different dosages of MWNTs.

TABLE 1 | Comparison of the tap-density of the two types of magnetic particles.

\begin{tabular}{lcc}
\hline Magnetic particles & $\begin{array}{c}\text { Tap-density } \boldsymbol{\rho} \\
\left(\mathbf{g} / \mathbf{c m}^{\mathbf{3}} \mathbf{)}\right.\end{array}$ & $\begin{array}{c}\text { Reduction } \\
\text { proportion (\%) }\end{array}$ \\
\hline Original Cl particles & 4.2 & - \\
Cl particles coated with MWNTs & 1.55 & 63.1 \\
\hline
\end{tabular}

\section{Surface Topography}

It is widely recognized that the quality of the coating effect can be reflected intuitively by the surface topography of particles. Therefore, the prepared CI particles coated with MWNTs, original CI particles and MWNTs are observed using the scanning electron microscope (SEM) with the resolution of $1 \mathrm{~nm}$, which can entirely present the surface topography characteristic of particles.

The electron micrographs of the original CI particles and MWNTs are as presented in Figure 4. Meanwhile, CI particles coated with MWNTs with different dosages of grafting agents named as A1-A3 with gradually increased dosages of graft agents, are observed, as shown in Figure 5. They show that the surfaces of the original CI particles are spherical and smooth, while become a little rough with some substances after coating. The coating effect increases with the increasing dosages of grafting agent within a certain range, while stabilizes or even decreases when beyond the limit. The coating layer of sample A1 is far from compacted

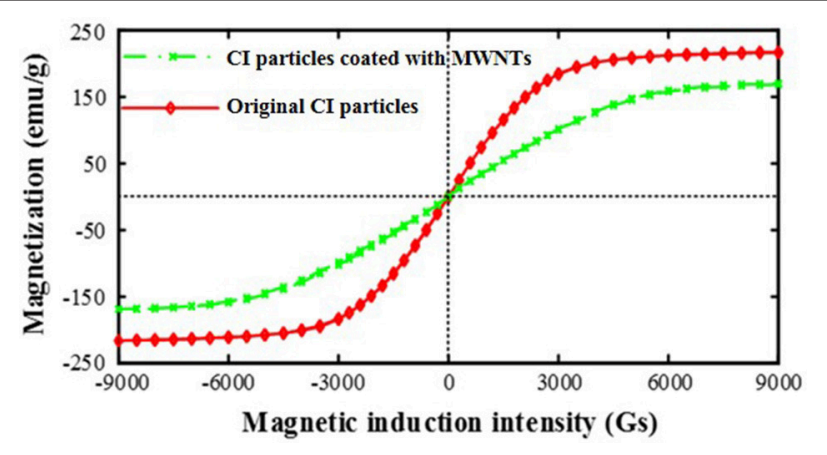

FIGURE 7 | Comparison of the magnetization curves of two kinds of the ferromagnetic particles ( $1 \mathrm{Gs}=0.0795775 \mathrm{kA} / \mathrm{m})$.

as only few filaments (MWNTs) are coated on the surface of the particles. As for sample A2, it is still difficult to coat on the surface of CI particles completely. While for sample A3, the filaments are relatively obvious through intertwined and spread out together in the spherical surface and almost all of the surfaces of CI particles are coated with MWNTs, which reflects that the dosages of grafting agent is enough. On the whole, the grafting agent is influential to the surface topography and the dosages of it should be paid great attention as it plays a major role in the quality of coating effect. And after comparison, the sample A3 
TABLE 2 | Comparison of the magnetic property of two types of magnetic particles.

\begin{tabular}{lcccc}
\hline Particles & $\begin{array}{c}\text { Saturation } \\
\text { magnetization } \\
\boldsymbol{M}_{\boldsymbol{s}}(\mathbf{e m u} / \mathbf{g})\end{array}$ & $\begin{array}{c}\text { Remanence } \\
\boldsymbol{M}_{\boldsymbol{r}}(\mathbf{e m u} / \mathbf{g})\end{array}$ & & \multicolumn{2}{c}{ Coercivity $\boldsymbol{H}_{\boldsymbol{C}}$} \\
\cline { 4 - 6 } & 217 & 0.1346 & 1.60 & 0.13 \\
\hline $\begin{array}{l}\text { Original Cl particles } \\
\text { Cl particles coated with } \\
\text { MWNTs }\end{array}$ & 170 & 0.1996 & 4.78 & 0.38 \\
\hline
\end{tabular}

TABLE 3 | Redispersibility observation of prepared MR fluids.

\begin{tabular}{lccc}
\hline Type & Samples & $\begin{array}{c}\text { Agglomeration } \\
\text { situation }\end{array}$ & Redispersibility \\
\hline $\begin{array}{l}\text { Different dosages of } \\
\text { grafting agents }\end{array}$ & A1 & Serious & Poor \\
& A2 & Slight & Good \\
& A3 & Slight & Good \\
\hline Different dosages of & B0 & Serious & Poor \\
MWNTs & B1 & few & Good \\
& B2 & Slight & Good \\
& B3 & Slight & Good \\
\hline
\end{tabular}

The dosages of grafting agent: $A 1<A 2<A 3$; the dosages of grafting MWNTs: $B 0<B 1<B 2<B 3$.

is considered to be the one with the best proportion of grafting agent in this step.

Additionally, the electron micrographs of CI particles coated with gradually increased dosages of MWNTs in the order of A3, B1, B2, and B3 are observed and as presented in Figure 6. It shows that the coating effect is improved at first and then decreased gradually with the sustained increase of MWNTs, and the coating affect reaches the optimal in sample B1 infested with compact and homogeneous coating layer filled with filaments. It can be interpreted from the coating mechanism that excessive MWNTs are more inclined to assemble rather than coated on the surface of CI particles during the self-assembly process, resulting in the aggregation of MWNTs up to pulling out the filaments on the surface of CI particles and destroying the coating layer. Therefore, the dosages of MWNTs should be controlled within a reasonable range to achieve a relatively satisfying coating effect. Besides, the sample B1 is regarded as the magnetic particle of $\mathrm{MR}$ fluids in this paper with its best coating effect.

\section{Particle Density}

Particle density is one of the direct factors that affect the sedimentation stability of MR fluids as the difference in density between the CI particles and the carrier liquid is the main cause of the sedimentation issue of MR fluids, specially, the smaller the density difference between CI particles and the carrier liquid is, the better the stability and lower sedimentation rate of MR fluids are.

The densities of the original CI particles and sample B1 are shown in Table 1, which are obtained by calculation using the
TABLE 4 | Sedimentation rate of MR fluids with different dosages of grafting agent.

\begin{tabular}{lcc}
\hline Samples & $\begin{array}{c}\text { Sedimentation rate after } \\
\mathbf{6 0} \text { days (\%) }\end{array}$ & $\begin{array}{c}\text { Sedimentation rate after } \\
\mathbf{9 0} \text { days }(\%)\end{array}$ \\
\hline A1 & 4.74 & 6.32 \\
A2 & 2.80 & 2.90 \\
A3 & 1.84 & 1.84 \\
\hline
\end{tabular}

The dosages of grafting agent: $A 1<A 2<A 3$.

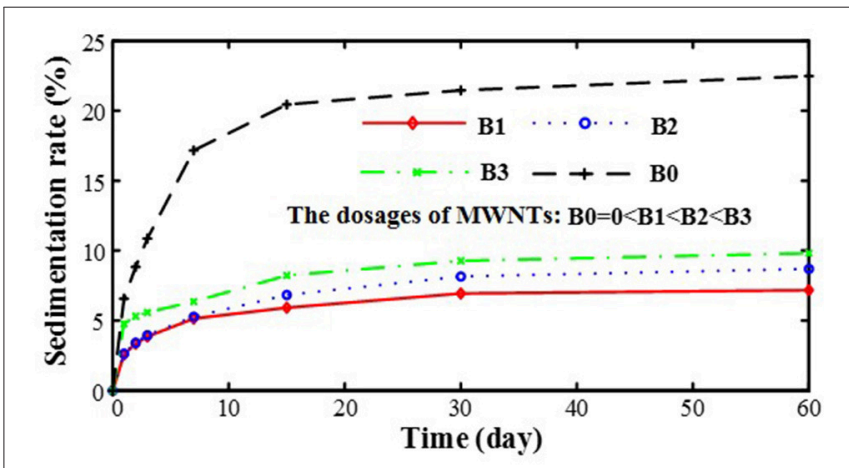

FIGURE 8 | Sedimentation rate of MR fluids with the different dosages of MWNTs.

volume and the mass gotten by respectively the cylinder and the electronic scale. It can be presented that the tap-density of sample $\mathrm{B} 1$ is greatly reduced compared with the original CI particles, which is from 4.2 to $1.55 \mathrm{~g} / \mathrm{cm}^{3}$ with a reduction of $63.1 \%$. It is obvious that the test results are consistent with the expectation as MWNTs is a kind of lightweight material that can reduce the density of the composite particles, which is favorable to improve the sedimentation stability of MR fluids.

\section{Magnetic Property}

Magnetic property is a significant index of the magnetic particles, which can directly affect the magnetic rheological property of MR fluids. Besides, the magnetic particles with better performance should have high saturation magnetization strength, less residual magnetism, and low coercive force simultaneously.

The magnetic property of particles is measured via vibration sample magnetometer with a maximum magnetic field of 1,600 $\mathrm{kA} / \mathrm{m}$, and the magnetic hysteresis curves of the two types of magnetic particles (CI particles coated with MWNTs (sample B1) and original CI particles) are shown in Figure 7. It can be concluded that the saturation magnetization intensity of sample B1 is slightly lower than that of the original CI particles under different magnetic field. This coincides with the fact that the magnetization intensity of MWNTs is much lower than that of the original CI particles that leads to the magnetization intensity of the composite particles reduced that. Additionally, further comparison of these two kinds of magnetic particles is shown in Table 2. It can be demonstrated that the saturation magnetization intensity of original CI particles is $217 \mathrm{emu} / \mathrm{g}$, while $170 \mathrm{emu} / \mathrm{g}$ 
for sample B1 with a reduction of $21.7 \%$. In terms of residual magnetism and coercive force, the sample B1 is a little higher than the original CI particles. In conclusion, although the magnetic property of CI particles coated with MWNTs is slightly reduced compared with the original CI particles, it is still superior to the other magnetic particles, such as hematite, magnetite and maghemite. Therefore, the CI particles coated with MWNTs are very appropriate for the preparation of MR fluids.

\section{Stability Tests of MR Fluids}

It is widely acknowledged that the stability of MR fluids mainly includes the redispersibility and the sedimentation stability. The redispersibility refers to the ability of an agglomerated MR fluids return to its origin state with excellent fluidity and homogeneity, which is closely correlated to the practical application of MR fluids. However, there is still no standardized evaluation system to evaluate it. Therefore, the natural observation method was adopted to test the redispersibility of MR fluids. After MR liquids are allowed to stand for a certain period of time, the agglomeration and redispersibility of MR fluids are observed by gently oscillating or shaking MR fluids. The observation results is as presented in Table 3. Table 3 shows that the redispersibility of sample B1 is the best.

Sedimentation stability is one of the most important properties of MR fluids which is evaluated by their sedimentation rates calculated by the following equation (Guo et al., 2017). The sedimentation ratio is defined as:

$$
\begin{aligned}
& \text { Sedimentation ratio }(\%)= \\
& \qquad \frac{\text { volume of the supernatant liquid }}{\text { volume of the entire mixture liquid }} \times 100 \%
\end{aligned}
$$

The sedimentation rates of MR fluids samples with gradual increase of dosages of grafting agent, which are named as A1-A3, are observed, and the results are as shown in Table 4. It can be presented that the sedimentation rate decreases with the increase of the dosages of grafting agent, i.e., the sedimentation rate of sample A1 is $4.74 \%$, while $2.80 \%$ for sample A2 even decreases to $1.84 \%$ for sample A3 after 60 days. It is obvious that the test results are consistent with the surface topography test when the dosages of grafting agent is within a certain range. The better of the coating affect is, the better of the sedimentation stability as the density of the composite particles is significantly decreased.

Additionally, the sedimentation rates of $\mathrm{MR}$ fluids with different dosages of MWNTs, which are named as B0-B3, are observed, as shown in Figure 8. Sample B0 is prepared based on the original CI particle, while samples B1, B2, and B3 are prepared based on the CI particles coated with MWNTs with increasing dosages of MWNTs. It can be inferred that the sedimentation stability of MR fluids is significantly improved because of the adaptation of MWNTs, the sedimentation rate of sample B0 is $22.47 \%$ after 60 days, which is the maximum value compared with other samples, representing that using CI particles coated with MWNTs can effectively reduce the sedimentation rates of MR fluids. On the other hand, the sedimentation rate of MR fluids increases with the increase of the dosages of MWNTs, i.e., the sedimentation rate of sample B1 is $7.18 \%$, while $9.79 \%$ for sample B3, which reflects that excessive MWNTs is unfavorable to the improvement of sedimentation stability of MR fluids cause the interaction between particles will be greatly affected because of the introduction of coating materials. Therefore, the dosages of MWNTs should be controlled in a rational range.

\section{CONCLUSION}

In this paper, MR fluids with CI particles coated with MWNTs were developed and the coating effect was studied through surface topography particle density, and magnetic properties of composite magnetic particles and stability tests of the prepared MR fluids. Meanwhile, the influence of the dosages of grafting agent and MWNTs on the coating effect were analyzed to verify the effect of CI particles coated with MWNTs on the improvement of the sedimentation stability. The conclusions are as the follows:

(1) The tests about the composite particles consisting of surface topography, particle density and magnetic property indicate that the composite particles with appropriate dosages of grafting agent and MWNTs will greatly benefit the coating effect, contributing to the implementation of composite particles with better performance. Moreover, the particle density decreased sharply while just a slightly decrease in magnetic property, which demonstrated that the coating material is favorable for the improvement of sedimentation stability while unfavorable for magnetism. Therefore, it is significant to balance the advantages and disadvantages caused by the introduction of coating material when the composite particles are prepared.

(2) The respersibility and the sedimentation tests of the prepared MR fluids show that the stability is greatly improved with the introduction of MWNTs because of the reduction in specific gravity. Meanwhile, the respersibility property and sedimentation rates of MR fluids are closely related to the coating effect of the composite particles, which is affected by the dosages of grafting agent and MWNTs.

\section{AUTHOR CONTRIBUTIONS}

Y-QG proposed the idea of this paper. Under the guidance of Y-QG, C-LS, and Z-DX, prepared MR fluids and finished the test of MR fluids. Y-QG, C-LS, and Z-DX jointly completed the writing of the article. XJ helped in proof reading of overall presentation and experimental data.

\section{FUNDING}

This study is financially supported by National Science Foundation for Distinguished Young Scholars of China (51625803), Yangtze River Scholars Distinguished Professor of Ministry of Education in China, Distinguished Professor of Jiangsu Province (Key Funding). Ten Thousand Talent Program (Innovation Leading Talents), and the Program for Jiangsu Province 333 Talents. These supports are gratefully acknowledged. 


\section{REFERENCES}

Arruebo, M., Fernandez-Pacheco, R., Ibarra, M. R., and Santamaría, J. (2007). Magnetic nanoparticles for drug delivery. Nano Today 2, 22-32. doi: 10.1016/S1748-0132(07)70084-1

Cheng, H. B., Wang, J. M., Zhang, Q. J., and Wereley, N. M. (2009). Preparation of composite magnetic particles and aqueous magnetorheological fluids. Smart Mater. Struct. 18:085009. doi: 10.1088/0964-1726/18/8/085009

Cho, M. S., Lim, S. T., Jang, I. B., Choi, H. J., and Jhon, M. S. (2004). Encapsulation of spherical iron-particle with PMMA and its magnetorheological particles. IEEE Trans. Magn. 40, 3036-3038. doi: 10.1109/TMAG.2004.830413

Dong, X. F., Ma, N., Qi, M., Li, J. H., Guan, X. C., and Ou, J. P. (2012). Properties of magneto-rheological fluids based on amorphous micro-particles. Trans. Nonferrous Metal. Soc. China 22, 2979-2983. doi: 10.1016/S1003-6326(11)61559-8

Du, C. B., Chen, W. Q., and Wan, F. X. (2010). Influence of HLB parameters of surfactants on properties of magneto-rheological fluid. Manufact. Sci. Eng. 97-101, 843-847. doi: 10.4028/www.scientific.net/AMR.97-101.843

Ferroudj, N., Nzimoto, J., Davidson, A., Talbot, D., Briot, E., Dupuis, V., et al. (2013). Maghemite nanoparticles and maghemite/silica nanocomposite microspheres as magnetic Fenton catalysts for the removal of water pollutants. Appl. Catal. B Environ. 136, 9-18. doi: 10.1016/j.apcatb.2013.01.046

Foister, R. T. (1997). Magnetorheological Fluids. U.S. Patent No 5667715. Washington, DC: U.S. Patent and Trademark Office.

Goldowsky, M. (1980). New methods for sealing, filtering and lubricating with magnetic fluids. IEEE Trans. Magn. 16, 382-386. doi: 10.1109/TMAG.1980.1060587

Guo, Y. Q., Xu, Z. D., Chen, B. B., Ran, C. S., and Guo, W. Y. (2017). Preparation and experimental study of magnetorheological fluid for vibration control. Int. J. Acoust. Vibrat. 22, 194-200. doi: 10.20855/ijav.2017. 22.2464

Jiang, W. Q., Zhu, H., Guo, C. Y., Li, J., Xue, Q., Feng, J., et al. (2010). Poly (methyl methacrylate)-coated carbonyl iron particles and their magnetorheological characteristics. Polym. Int. 59, 879-883. doi: 10.1002/pi.2794

Kim, H. J., Kim, G. C., Lee, G. S., Hong, M. T., and Choi, H. J. (2013). Viscosity of magnetorheological fluids using iron-silicon nanoparticles. J. Nanosci. Nanotechnol. 13, 6005-6009. doi: 10.1166/jnn.2013.7638

Kim, J. E., and Choi, H. J. (2011). Magnetic carbonyl iron particle dispersed in viscoelastic fluid and its magnetorheological property. IEEE Trans. Magn. 47, 3173-3176. doi: 10.1109/TMAG.2011.2156396

Kim, Y. S., and Kim, Y. H. (2003). Application of ferro-cobalt magnetic fluid for oil sealing. J. Magn. Magn. Mater. 267, 105-110. doi: 10.1016/S0304-8853(03)00342-1

Kormann, C., Laun, H. M., and Richter, H. J. (1996). MR fluids with nano-sized magnetic particles. Int. J. Modern Phys. B 10, 3167-3172. doi: 10.1142/S0217979296001604

Liu, Y. D., Hong, C. H., and Choi, H. J. (2012). Polymeric colloidal magnetic composite microspheres and their magneto-responsive characteristics. Macromol. Res. 20, 1211-1218. doi: 10.1007/s13233-012-0198-8

Lopez-Lopez, M. T., Kuzhir, P., Caballero-Hernandez, J., Rodríguez-Arco, L., Duran, J. D. G., and Bossis, G. (2012). Yield stress in magneto- rheological suspensions near the limit of maximum-packing fraction. J. Rheol. 56, 1209-1224. doi: 10.1122/1.4731659

Mangal, S. K., and Kumar, A. (2015). Geometric parameter optimization of magneto-rheological damper using design of experiment technique. Int. J. Mech. Mater. Eng. 10:4. doi: 10.1186/s40712-015-0031-1
Mitamura, Y., Arioka, S., Sakota, D., Sekine, K., and Azegami, M. (2008). Application of a magnetic fluid seal to rotary blood pumps. J. Phys. Condens. Matter 20:204145. doi: 10.1088/0953-8984/20/20/204145

Mrlik, M., Ilcikova, M., Pavlinek, V., Mosnáček, J., Peer, P., and Filip, P. (2013). Improved thermooxidation and sedimentation stability of covalently-coated carbonyl iron particles with cholesteryl groups and their influence on magnetorheology. J. Colloid Interface Sci. 396, 146-151. doi: 10.1016/j.jcis.2013.01.027

Oh, J. K., and Park, J. M. (2011). Iron oxide-based superparamagnetic polymeric nanomaterials: design, preparation, and biomedical application. Prog. Polym. Sci. 36, 168-189. doi: 10.1016/j.progpolymsci.2010.08.005

Olabi, A. G., and Grunwald, A. (2007). Design and application of magnetorheological fluid. Mater. Des. 28, 2658-2664. doi: 10.1016/j.matdes.2006.10.009

Qiao, X. Y., Bai, M. W., Tao, K., Gong, X., Gu, R., Watanabe, H., et al. (2010). Magnetorheological behavior of polyethyene glycol-coated $\mathrm{Fe}_{3} \mathrm{O}_{4}$ ferrofluids. Nihon Reoroji Gakkaishi 38, 23-30. doi: 10.1678/rheology.38.23

Rabinow, J. (1948). The magnetic fluid clutch. Trans. Am. Inst. Electrical Eng. 67, 1308-1315. doi: 10.1109/T-AIEE.1948.5059821

Sedlacik, M., Pavlinek, V., Lehocky, M., Mracek, A., Grulich, O., Svrcinova, P., et al. (2011). Plasma-treated carbonyl iron particles as a dispersed phase in magnetorheological fluids. Colloid. Surf. A Physicochem. Eng. Aspects 387, 99-103. doi: 10.1016/j.colsurfa.2011.07.035

Sutrisno, J., Fuchs, A., Sahin, H., and Gordaninejad, F. (2013). Surface coated iron particles via atom transfer radical polymerization for thermal-oxidatively stable high viscosity magnetorheological fluid. J. Appl. Polym. Sci. 128, 470-480. doi: 10.1002/app.38199

Thomas, J. R. (1966). Preparation and magnetic properties of colloidal cobalt particles. J. Appl. Phys. 37, 2914-2915. doi: 10.1063/1.1782154

Tian, Z. Z., Chen, F., and Wu, X. F. (2016). A novel preparation process for magnetorheological fluid with high sedimentation stability. Mater. Manufact. Process. 31, 2030-2036. doi: 10.1080/10426914.2016.11 98032

Ulicny, J. C., and Mance, A. M. (2004). Evaluation of electroless nickel surface treatment for iron powder used in MR fluids. Mater. Sci. Eng. A. 369, 309-313. doi: 10.1016/j.msea.2003.11.039

Xu, Z. D., Shen, Y. P., and Guo, Y. Q. (2003). Semi-active control of structures incorporated with magnetorheological dampers using neural networks. Smart Mater. Struct. 12, 80-87. doi: 10.1088/0964-1726/12/1/309

Zhu, M., Yu, M., Qi, S., and Fu, J. (2018). Investigations on response time of magnetorheological elastomer under compression mode. Smart Mater. Struct. 27:055017. doi: 10.1088/1361-665X/aab63e

Zong, L. H., Gong, X. L., Xuan, S. H., and Guo, C. Y. (2013). Semi-active H infinity control of high-speed railway vehicle suspension with magnetorheological dampers. Vehicle Syst. Dyn. 51, 600-626. doi: 10.1080/00423114.2012.7 58858

Conflict of Interest Statement: The authors declare that the research was conducted in the absence of any commercial or financial relationships that could be construed as a potential conflict of interest.

Copyright (C) 2018 Guo, Sun, Xu and Jing. This is an open-access article distributed under the terms of the Creative Commons Attribution License (CC BY). The use, distribution or reproduction in other forums is permitted, provided the original author(s) and the copyright owner(s) are credited and that the original publication in this journal is cited, in accordance with accepted academic practice. No use, distribution or reproduction is permitted which does not comply with these terms. 